\title{
Ajuste vegetativo-productivo a través del despampanado en floración: Efectos agronómicos y cualitativos en cv. Verdejo en la D.O. Rueda
}

\section{Vegetative-productive adjustment through shoot removal at flowering: Agronomic and qualitative effects on cv. Verdejo, in the D.O. Rueda (Spain)}

\author{
J. Yuste y D. Martínez-Porro \\ Instituto Tecnológico Agrario de Castilla y León, 47071 Valladolid, España
}

Resumen. El ajuste del desarrollo vegetativo y la carga productiva puede ser un aspecto crítico para aproximarse al equilibrio del viñedo y mejorar la calidad de la uva en variedades blancas, dependiendo, entre otros factores, de las condiciones hídricas de cultivo. La variedad Verdejo está extendiendo intensamente su viñedo en diversas zonas, por lo que conviene conocer su respuesta a la reducción de su expresión vegetativa con el fin de favorecer la calidad de la uva.

A lo largo del período 2015-2017, se estudió la aplicación de un tratamiento de despampanado (D), suprimiendo 6 pámpanos de los 16 de cada cepa (dejando 10 por cepa), frente a un tratamiento testigo (T), en régimen hídrico deficitario: riego del $30 \%$ de ETo aplicado por goteo semanalmente desde el inicio del envero hasta la vendimia. El ensayo, ubicado en Medina del Campo (Valladolid), dentro de la D.O. Rueda, se llevó a cabo con cv. Verdejo sobre portainjerto 110R, plantado en 2006 y conducido en espaldera en cordón Royat bilateral, con un marco de $2.60 \mathrm{~m} \times 1.25 \mathrm{~m}$ (3.077 cepas/ha).

Las cepas sometidas a despampanado redujeron un $16 \%$ el peso de madera de poda, a pesar del aumento del peso del sarmiento. Asimismo, el despampanado redujo un $32 \%$ la producción de uva, como consecuencia del descenso en el número de racimos. El despampanado favoreció la concentración de azúcares, así como, en menor cuantía, el pH y la concentración de potasio, mientras que la acidez total y el ácido tartárico no presentaron una tendencia interanual definida ni constante, y el ácido málico mostró valores anuales con tendencia inversa entre tratamientos con respecto al ácido tartárico, sin diferencias notables entre tratamientos. Estos resultados se corresponden con cierta intensificación del proceso de maduración de la uva, causada por el aumento del ratio hojas/frutos debido al despampanado. En definitiva, se vislumbra la posible utilidad del despampanado en añadas en que pueda haber retraso en el proceso de maduración, con el fin de optimizar la fecha de vendimia y la calidad de la uva, según los objetivos perseguidos.

\begin{abstract}
The adjustment of vegetative development and grape load can be a critical aspect for approaching to the vine balance and improve the grape quality of white grape varieties, depending, among other factors, on the culture water conditions in areas of serious rainfall limitations, as it happens in most of the Spanish growing regions. The vineyards of Verdejo variety are spreading intensely in different zones, so that it is convenient to know its response to the reduction of its vegetative expression in order to favour the quality of grape and wine. During the period 2015-2017, the application of shoot removal (D) was studied, by means of pulling out $37.5 \%$ of shoots (from 16 to keep 10 per vine) in comparison with a control treatment (T). The vineyard was grown under deficitary water regime: drip irrigation of 30\% ETo applied once a week from veraison until harvest. The trial was developed with vines of cv. Verdejo on 110R rootstock, planted in 2006 and trellised as a Royat bilateral cordon. The vine distances were $2.60 \mathrm{~m} \times 1.25 \mathrm{~m}$ (3.077 plants/ha). The vineyard was located in Medina del Campo (Valladolid, Spain), belonging to the D.O. Rueda. The vines of Verdejo submitted to shoot removal reduce its final vegetative growth, shown by a clear decrease of the wood pruning weight, in spite of the increase of the individual shoot weight. Likewise, the shoot removal caused a remarkable reduction of grape yield, due to the reduction of the number of clusters. Related to the grape quality, it favoured the sugar concentration, as well as, to a lesser extent, in a more variable way, the titratable acidity, the tartaric acid, the $\mathrm{pH}$ and the potassium concentration. The malic acid was generally reduced because of the deshooting, although it also varied according to the year. To sum up, it may be worth highlighting the usefulness of the shoot removal to improve the ripening of the grape, through the vegetative and productive reduction, depending on the annual weather conditions.
\end{abstract}




\section{Introducción}

El nivel de rendimiento puede ser un aspecto crítico para la calidad de la uva, sobre todo en condiciones de cultivo con fuerte limitación hídrica, como las que caracterizan a la mayoría de regiones en España, y se ha observado en la variedad tinta Tempranillo en diversas situaciones [1]. La incidencia del nivel productivo en la calidad de la uva ha sido menos estudiada en variedades blancas, si bien algunos trabajos relativos al control del rendimiento en la variedad Verdejo apuntan ciertos efectos en distintas situaciones hídricas [2,3].

La técnica básica inicial para acercarse al equilibrio vegetativo-productivo requerido para la calidad de la uva es la poda de invierno, a través de la cual se establece una carga de yemas francas cuyo número dependerá de la variedad y del medio, teniendo en cuenta los objetivos de producción y calidad perseguidos en cada viñedo [4]. Sin embargo, las circunstancias medioambientales anuales pueden ocasionar un número excesivo de pámpanos con respecto a las yemas dejadas en la poda o una fertilidad real mayor de la esperada [3]. En dicha situación, para ejercer un control inmediato del rendimiento suele emplearse la técnica del aclareo de racimos, puesto que es conocido que el incremento inicial de producción es acompañado por un incremento en la calidad de la uva, mostrando la regresión entre estos dos parámetros una curva normal, hasta un punto a partir del cual la calidad se resiente debido al exceso de carga de cosecha [5]. Este punto de equilibrio dependerá de la capacidad productiva del viñedo, que será función del clima, el suelo, la variedad, el manejo del cultivo y el objetivo productivo deseado [6].

La reducción de cosecha modifica la relación fuentesumidero y puede afectar al proceso de maduración de la uva según la variedad [7]. Así, Intrigliolo y Castell expusieron que el aclareo de racimos incrementa la uniformidad en cuanto a sólidos solubles [8]. Contrariamente, Calderón-Orellana et al. escribieron que el aclareo de racimos no mantuvo la uniformidad del contenido en sólidos solubles hasta vendimia con respecto al testigo [9]. No obstante, ambas publicaciones coinciden en que los cambios debidos al aclareo de racimos, en la composición de la uva y las características del vino resultante, pueden verse modificados en diverso grado en función de las características climáticas del año.

La relación fuente-sumidero puede alcanzarse con distinto nivel de expresión vegetativa, el cual vendrá condicionado por la carga de poda. La calidad de uva máxima que puede ser alcanzada disminuye al aumentar la expresión vegetativa, aunque a medida que aumenta el nivel de expresión vegetativa el máximo de calidad, dentro de ella, se corresponde con una cosecha mayor [6].

Diversas estrategias de poda en seco han sido probadas, en viñedos maduros, para tratar de reducir la expresión vegetativa del viñedo $[10,11]$, pero su capacidad de retención depende enormemente del medio. Por este motivo, el ajuste de pámpanos en la fase de desarrollo de las inflorescencias se vislumbra como un método eficaz para reducir el exceso de vigor y limitar el nivel de expresión vegetativa [11]. La capacidad que el despampanado pueda tener para modificar el desarrollo vegetativo-productivo y la calidad de la uva dependerá de los diversos factores que condicionan el cultivo del viñedo, tales como el suelo, el clima, el tipo de manejo y la variedad [12]. Esto suscita el interés por la experimentación en cada zona con aquella o aquellas variedades que pueden ser susceptibles de un control de la producción para mejorar la calidad de la uva.

El objeto de este trabajo es el estudio de los posibles efectos de la aplicación del despampanando en la época de inicio de floración, en el desarrollo vegetativo y en la producción de uva, así como en su calidad, de la variedad Verdejo, injertada sobre 110R, y conducida en espaldera, a lo largo de un periodo de tres años, en un ensayo experimental situado en la Denominación de Origen Rueda (España), por tanto, en las condiciones climáticas típicas del centro del valle del río Duero.

\section{Material y métodos}

El ensayo se realizó a lo largo del periodo 20152017 en la localidad de Medina del Campo (Valladolid, España), en un viñedo perteneciente a la bodega del Grupo Yllera S.L., ubicado dentro de la D.O. Rueda, en el centro de Castilla y León. Las coordenadas del ensayo son $41^{\circ} 21^{\prime} 02^{\prime \prime} \mathrm{N}$ y $4^{\circ} 56^{\prime} 16^{\prime \prime} \mathrm{O}$. Las cepas del viñedo experimental, plantadas en 2006, son del cv. Verdejo, injertadas sobre el portainjerto 110R.

Las cepas han sido conducidas en espaldera, con formación en cordón Royat bilateral y posicionamiento vertical de la vegetación (VSP), cuya orientación de filas es NNO $\left(\mathrm{N}-25^{\circ}\right)$. La carga de poda fue de 16 yemas por cepa, en pulgares de 2 yemas. Se aplicó una operación de poda en verde cada año, tras el período de riesgo de helada primaveral, para ajustar la carga de pámpanos por cepa a la carga de yemas francas establecida en la poda de invierno.

Los tratamientos experimentales se basan en la regulación del número de pámpanos, a través de la aplicación de un tratamiento de despampanado (D) frente a un tratamiento testigo (T). El despampanado se aplica al inicio de la floración, mediante una reducción de 16 a 10 pámpanos por cepa (37.5\%).

El marco de plantación es de $2.60 \mathrm{~m} \times 1.25 \mathrm{~m}$, que corresponde a un área de suelo por cepa de $3.25 \mathrm{~m}^{2}$, o sea, a una densidad de 3.077 cepas por ha. El diseño experimental es en bloques al azar, con 4 repeticiones de 20 cepas de control por parcela elemental.

El suelo del ensayo es profundo, con un horizonte superior de textura arcillosa, y presenta una suave pendiente en dirección Este-Oeste y un buen drenaje en general.

El régimen hídrico de cultivo del viñedo consistió en la aplicación de riego del $30 \%$ de ETo desde el estado de fenológico de tamaño guisante hasta vendimia, lo que se tradujo en 66, 73 y $74 \mathrm{~mm}$ en 2015, 2016 y 2017 respectivamente.

Se determinaron parámetros de desarrollo vegetativo, rendimiento y composición de la uva, como se refleja en el apartado de resultados.

El análisis estadístico de los resultados se ha realizado mediante análisis de varianza (ANOVA), con el programa SPSS 16.0.

Los datos medios mensuales de pluviometría y temperatura, correspondientes al periodo 2015-2017, se detallan en las Tablas 1 a 3. 
Tabla 1. Datos termo-pluviométricos medios de la campaña 2015 (octubre-2014 / septiembre-2015), registrados en Medina del Campo (Valladolid). $T_{\mathrm{m}}$ : temperatura media $\left({ }^{\circ} \mathrm{C}\right), T_{\max }$ : temperatura media de máximas $\left({ }^{\circ} \mathrm{C}\right), T_{\min }$ : temperatura media de mínimas $\left({ }^{\circ} \mathrm{C}\right)$, P: precipitación $(\mathrm{mm})$, para todas las tablas.

\begin{tabular}{|lllcccll|}
\hline & Oct & Nov & Dic & Ene & Feb & Mar & \\
\hline$T_{\mathrm{m}}$ & 15.7 & 9.4 & 3.7 & 1.6 & 4.1 & 8.6 & \\
\hline$T_{\max }$ & 22.7 & 13.4 & 7.8 & 6.5 & 9.2 & 15.7 & \\
\hline$T_{\min }$ & 10.2 & 5.9 & 0.2 & -2.3 & -0.2 & 1.7 & \\
\hline $\mathbf{P}$ & 14.1 & 47.4 & 15.9 & 25.7 & 10.3 & 4.6 & \\
\hline & $\mathbf{A b r}$ & May & Jun & Jul & Ago & Sep & Año \\
\hline$T_{\mathrm{m}}$ & 11.9 & 16.4 & 20.7 & 24.4 & 21.7 & 16.6 & 12.9 \\
\hline$T_{\max }$ & 18.4 & 24.0 & 28.5 & 32.9 & 29.8 & 24.4 & 19.4 \\
\hline$T_{\min }$ & 5.8 & 8.3 & 13.2 & 15.8 & 13.9 & 9.5 & 6.8 \\
\hline $\mathbf{P}$ & 43.5 & 10.1 & 46.9 & 3.9 & 6.8 & 11.1 & 240.3 \\
\hline
\end{tabular}

Tabla 2. Datos termo-pluviométricos de la campaña 2016 (octubre-2015 / septiembre-2016), registrados en Medina del Campo (Valladolid)

\begin{tabular}{|llllccll|}
\hline & Oct & Nov & Dic & Ene & Feb & Mar & \\
\hline$T_{\mathrm{m}}$ & 15.7 & 9.4 & 3.7 & 1.6 & 4.1 & 8.6 & \\
\hline$T_{\max }$ & 22.7 & 13.4 & 7.8 & 6.5 & 9.2 & 15.7 & \\
\hline$T_{\min }$ & 10.2 & 5.9 & 0.2 & -2.3 & -0.2 & 1,7 & \\
\hline $\mathbf{P}$ & 14.1 & 47.4 & 15.9 & 25.7 & 10.3 & 4.6 & \\
\hline & Abr & May & Jun & Jul & Ago & Sep & Año \\
\hline$T_{\mathrm{m}}$ & 9.3 & 13.7 & 19.7 & 23.5 & 22.9 & 19.1 & 12.7 \\
\hline$T_{\max }$ & 15.1 & 19.9 & 27.3 & 32.0 & 31.5 & 27.2 & 19.1 \\
\hline$T_{\min }$ & 4.2 & 7.8 & 11.6 & 14.9 & 14.2 & 11.2 & 6.9 \\
\hline $\mathbf{P}$ & 44.5 & 48.7 & 0.1 & 0.1 & 0.0 & 0.0 & 317.6 \\
\hline
\end{tabular}

Tabla 3. Datos termo-pluviométricos de la campaña 2017 (octubre-2016 / septiembre-2017), registrados en Medina del Campo (Valladolid)

\begin{tabular}{|llllclll|}
\hline & Oct & Nov & Dic & Ene & Feb & Mar & \\
\hline$T_{\mathrm{m}}$ & 13.7 & 7.2 & 3.9 & 2.7 & 7.0 & 9.1 & \\
\hline$T_{\max }$ & 21.1 & 12.2 & 7.8 & 9.2 & 12.7 & 16.4 & \\
\hline$T_{\min }$ & 7.6 & 2.6 & 1.0 & -2.4 & 2.0 & 2.4 & \\
\hline $\mathbf{P}$ & 18.1 & 33.0 & 11.5 & 3.1 & 12.1 & 0.6 & \\
\hline & $\mathbf{A b r}$ & May & Jun & Jul & Ago & Sep & Año \\
\hline$T_{\mathrm{m}}$ & 12.9 & 17.0 & 22.7 & 22.6 & 22.1 & 17.6 & 13.2 \\
\hline$T_{\max }$ & 21.2 & 24.3 & 30.7 & 30.7 & 30.7 & 26.0 & 20.3 \\
\hline$T_{\min }$ & 4.1 & 9.2 & 14.4 & 14.0 & 13.3 & 9.3 & 6.5 \\
\hline $\mathbf{P}$ & 0.7 & 39.8 & 5.5 & 31.3 & 11.7 & 0.0 & 167.4 \\
\hline
\end{tabular}

Tabla 4. Rendimiento (t/ha). Significación estadística $*=p<5 \%$ (para todas las tablas).

\begin{tabular}{|lllll|}
\hline Trat. & $\mathbf{2 0 1 5}$ & $\mathbf{2 0 1 6}$ & $\mathbf{2 0 1 7}$ & Media \\
\hline T & 11.16 & 13.58 & 7.64 & 10.79 \\
\hline D & 7.97 & 9.04 & 4.84 & 7.28 \\
\hline Sig. & $*$ & $*$ & $*$ \\
\hline
\end{tabular}

\section{Resultados y discusión}

\subsection{Producción de uva}

El rendimiento en uva del tratamiento sometido a despampanado mostró una reducción notable con respecto al testigo, resultando un valor promedio de dicho tratamiento aproximadamente un $32 \%$ inferior al del testigo. El porcentaje de reducción fue muy constante a lo largo de los años a pesar de la clara variación del nivel productivo entre ellos, resultando las diferencias
Tabla 5. Peso de racimo $(\mathrm{g})$.

\begin{tabular}{|lllll|}
\hline Trat. & $\mathbf{2 0 1 5}$ & $\mathbf{2 0 1 6}$ & $\mathbf{2 0 1 7}$ & Media \\
\hline T & 144 & 162 & 96 & 134 \\
\hline D & 144 & 158 & 90 & 131 \\
\hline Sig. & & & & \\
\hline
\end{tabular}

Tabla 6. Racimos / cepa.

\begin{tabular}{|lllll|}
\hline Trat. & $\mathbf{2 0 1 5}$ & $\mathbf{2 0 1 6}$ & $\mathbf{2 0 1 7}$ & Media \\
\hline T & 25.1 & 26.9 & 25.8 & 25.9 \\
\hline D & 17.9 & 18.5 & 17.6 & 18.0 \\
\hline Sig. & $*$ & $*$ & $*$ & \\
\hline
\end{tabular}

Tabla 7. Bayas / racimo.

\begin{tabular}{|lllll|}
\hline Trat. & $\mathbf{2 0 1 5}$ & $\mathbf{2 0 1 6}$ & $\mathbf{2 0 1 7}$ & Media \\
\hline T & 110 & 132 & 76 & 106 \\
\hline D & 105 & 117 & 75 & 99 \\
\hline Sig. & & & & \\
\hline
\end{tabular}

Tabla 8. Peso de baya (g).

\begin{tabular}{|lllll|}
\hline Trat. & $\mathbf{2 0 1 5}$ & $\mathbf{2 0 1 6}$ & $\mathbf{2 0 1 7}$ & Media \\
\hline T & 1.31 & 1.22 & 1.27 & 1.27 \\
\hline D & 1.37 & 1.34 & 1.19 & 1.30 \\
\hline Sig. & & & & \\
\hline
\end{tabular}

Tabla 9. Racimos / sarmiento.

\begin{tabular}{|lllll|}
\hline Trat. & $\mathbf{2 0 1 5}$ & $\mathbf{2 0 1 6}$ & $\mathbf{2 0 1 7}$ & Media \\
\hline T & 1.53 & 1.72 & 1.72 & 1.66 \\
\hline D & 1.54 & 1.80 & 1.66 & 1.67 \\
\hline Sig. & & & & \\
\hline
\end{tabular}

estadísticamente significativas cada año (Tabla 4). El peso del racimo no mostró ningún efecto causado por el tratamiento de despampanado, por lo que los resultados no advirtieron diferencias estadísticamente significativas entre tratamientos (Tabla 5). El número de racimos por cepa explicaría completamente la reducción en la producción de uva causada por el despampanado, ya que dicho número se vio reducido directamente por la eliminación de pámpanos en floración, en un $31 \%$ de media interanual con respecto al testigo (Tabla 6).

En relación con el peso del racimo, el número de bayas por racimo mostró una ligera tendencia, aunque no constante, del tratamiento de despampanado a presentar valores ligeramente inferiores que el testigo, con una disminución media del 6\% (Tabla 7), no habiéndose encontrado diferencias estadísticamente significativas entre tratamientos. En cuanto al peso de baya, apenas se observó una escasa tendencia del tratamiento de despampanado a producir bayas de tamaño ligeramente mayor, con un valor medio de $1.30 \mathrm{~g}$ frente a $1.27 \mathrm{~g}$ de peso correspondiente al tratamiento testigo, sin que las diferencias anuales resultasen estadísticamente significativas (Tabla 8).

La fertilidad real, expresada como número de racimos por sarmiento, no mostró una tendencia definida entre tratamientos, resultando una media interanual similar en ambos, sin que las pequeñas diferencias anuales observadas resultasen estadísticamente significativas (Tabla 9). 
Tabla 10. Madera de poda (t/ha). Significación estadística $*=\mathrm{p}<5 \%$ (para todas las tablas).

\begin{tabular}{|lllll|}
\hline Trat. & $\mathbf{2 0 1 5}$ & $\mathbf{2 0 1 6}$ & $\mathbf{2 0 1 7}$ & Media \\
\hline T & 1.85 & 2.17 & 1.32 & 1.78 \\
\hline D & 1.67 & 1.79 & 1.01 & 1.49 \\
\hline Sig. & $*$ & $*$ & $*$ & \\
\hline
\end{tabular}

Tabla 11. Sarmientos / cepa.

\begin{tabular}{|lllll|}
\hline Trat. & $\mathbf{2 0 1 5}$ & $\mathbf{2 0 1 6}$ & $\mathbf{2 0 1 7}$ & Media \\
\hline T & 16.4 & 15.6 & 15.0 & 15.7 \\
\hline D & 11.6 & 10.3 & 10.6 & 10.8 \\
\hline Sig. & $*$ & $*$ & $*$ & \\
\hline
\end{tabular}

Tabla 12. Peso de sarmiento $(\mathrm{g})$.

\begin{tabular}{|lllll|}
\hline Trat. & $\mathbf{2 0 1 5}$ & $\mathbf{2 0 1 6}$ & $\mathbf{2 0 1 7}$ & Media \\
\hline T & 36.6 & 45.1 & 28.6 & 36.8 \\
\hline D & 46.6 & 56.5 & 31.6 & 44.9 \\
\hline Sig. & $*$ & $*$ & & \\
\hline
\end{tabular}

Tabla 13. Indice de Ravaz.

\begin{tabular}{|lllll|}
\hline Trat. & $\mathbf{2 0 1 5}$ & $\mathbf{2 0 1 6}$ & $\mathbf{2 0 1 7}$ & Media \\
\hline T & 6.08 & 6.25 & 5.86 & 6.06 \\
\hline D & 4.82 & 5.02 & 5.26 & 5.03 \\
\hline Sig. & $*$ & $*$ & & \\
\hline
\end{tabular}

\subsection{Desarrollo vegetativo}

El peso de madera de poda por cepa, y en consecuencia por unidad de superficie de suelo, se vio claramente modificado por la reducción del número de pámpanos, mostrando un valor medio, a lo largo del periodo de estudio, netamente inferior (en torno al 16\%) en el despampanado que en el testigo. La reducción observada en el peso de madera de poda del tratamiento de despampanado varió en función del año de estudio, aumentando cada año, con diferencias estadísticamente significativas anuales respecto al testigo (Tabla 10).

El número de sarmientos por cepa se redujo significativamente en el tratamiento de despampanado, en torno a un $31 \%$ de media interanual, lógicamente como consecuencia de la eliminación directa de pámpanos, en la que se respetaron 10 en cada cepa, frente a los 16 teóricos del tratamiento testigo, cada año al inicio de la floración (Tabla 11). Esta reducción del número de pámpanos fue la causa fundamental de la disminución de madera de poda en el tratamiento de despampanado.

El peso de sarmiento se vio favorecido por la aplicación del despampanado, con un valor medio aproximado de $37 \mathrm{~g}$ para el tratamiento testigo y $45 \mathrm{~g}$ para el tratamiento de despampanado (Tabla 12), que representa un aumento del $22 \%$. Este aumento compensó parcial, pero notoriamente, la reducción del número de pámpanos en cuanto al peso final de madera de poda, con diferencias estadísticamente significativas en 2015 y 2016.

El índice de Ravaz mostró una reducción derivada de la mayor disminución de la producción de uva que del peso de madera de poda en el tratamiento sometido a despampanado, habiéndose observado diferencias estadísticamente significativas en 2015 y 2016 (Tabla 13).
Tabla 14. Azúcares ( ${ }^{\circ}$ brix). Significación estadística $*=\mathrm{p}<5 \%$ (para todas las tablas).

\begin{tabular}{|lllll|}
\hline Trat. & $\mathbf{2 0 1 5}$ & $\mathbf{2 0 1 6}$ & $\mathbf{2 0 1 7}$ & Media \\
\hline T & 22.7 & 21.7 & 22.5 & 22.3 \\
\hline D & 23.4 & 22.4 & 23.3 & 23.0 \\
\hline Sig. & $*$ & $*$ & $*$ & \\
\hline
\end{tabular}

Tabla 15. Valor de $\mathrm{pH}$.

\begin{tabular}{|lllll|}
\hline Trat. & $\mathbf{2 0 1 5}$ & $\mathbf{2 0 1 6}$ & $\mathbf{2 0 1 7}$ & Media \\
\hline T & 3.36 & 3.30 & 3.37 & 3.34 \\
\hline D & 3.39 & 3.38 & 3.37 & 3.38 \\
\hline Sig. & $*$ & $*$ & & \\
\hline
\end{tabular}

\subsection{Composición de la uva}

La concentración de sólidos solubles $\left({ }^{\circ}\right.$ brix) se incrementó a favor del tratamiento de despampanado todos los años, resultando un aumento medio de $0.7^{\circ}$ brix respecto al testigo, habiéndose encontrado diferencias estadísticamente significativas todos los años (Tabla 14). Esta respuesta de las plantas está relacionada con el índice de Ravaz observado, pues el tratamiento de despampanado dispuso de una relación hojas a frutos más favorable que el testigo a lo largo del ciclo estival. En este mismo sentido, los años menos productivos, el primero y el tercero, mostraron mayor concentración de azúcares que el más productivo, el segundo.

Los valores de $\mathrm{pH}$ variaron ligeramente a favor del tratamiento de despampanado, excepto en el último año, de mayor sequía, habiéndose observado diferencias estadísticamente significativas, respecto al testigo, los dos primeros años (Tabla 15).

La acidez total $\left(\mathrm{g} / \mathrm{L} \mathrm{TH}_{2}\right)$ no presentó una tendencia constante ni notable entre tratamientos, con valores más altos del despampanado el primer y el último año, y más bajo el segundo año, el más productivo, que el testigo. Las diferencias anuales entre tratamientos no resultaron estadísticamente significativas (Tabla 16).

El ácido tartárico (g/L) presentó una tendencia interanual entre tratamientos similar a la acidez total, con un ligero aumento de valores en el tratamiento de despampanado el primer y el tercer año y una reducción clara en el segundo año, con respecto al testigo. Las diferencias anuales resultaron estadísticamente significativas el segundo y el tercer año, pero en sentido inverso entre ambos tratamientos (Tabla 17).

El ácido málico (g/L) mostró una tendencia entre tratamientos inversa al ácido tartárico, reduciéndose ligeramente con el despampanado el primer y el tercer año y aumentando más claramente el segundo año, más productivo, con respecto al testigo, aunque las diferencias entre tratamientos no resultaron estadísticamente significativas (Tabla 18).

La concentración de potasio $(\mathrm{mg} / \mathrm{L})$ fue ligeramente superior todos los años, más en el primero, en el tratamiento de despampanado que en el testigo, pero las diferencias sólo resultaron estadísticamente significativas el primer año (Tabla 19). Este resultado se corresponde con cierta intensificación del proceso de maduración de la uva, como se ve reflejada en los azúcares y el pH, causada por la modificación del ratio hojas/frutos debida al despampanado. 
Tabla 16. Acidez total $\left(\mathrm{g} / \mathrm{L} \mathrm{TH}_{2}\right)$.

\begin{tabular}{|lllll|}
\hline Trat. & $\mathbf{2 0 1 5}$ & $\mathbf{2 0 1 6}$ & $\mathbf{2 0 1 7}$ & Media \\
\hline T & 5.08 & 5.87 & 5.82 & 5.59 \\
\hline D & 5.14 & 5.51 & 6.02 & 5.56 \\
\hline Sig. & & & & \\
\hline
\end{tabular}

Tabla 17. Acido tartárico (g/L).

\begin{tabular}{|lllll|}
\hline Trat. & $\mathbf{2 0 1 5}$ & $\mathbf{2 0 1 6}$ & $\mathbf{2 0 1 7}$ & Media \\
\hline T & 8.28 & 10.12 & 9.73 & 9.38 \\
\hline D & 8.48 & 9.26 & 10.30 & 9.35 \\
\hline Sig. & \multicolumn{3}{c}{$*$} \\
\hline
\end{tabular}

Tabla 18. Acido tartárico (g/L).

\begin{tabular}{|lllll|}
\hline Trat. & $\mathbf{2 0 1 5}$ & $\mathbf{2 0 1 6}$ & $\mathbf{2 0 1 7}$ & Media \\
\hline T & 0.85 & 0.97 & 1.16 & 0.99 \\
\hline D & 0.76 & 1.22 & 1.07 & 1.02 \\
\hline Sig. & & & & \\
\hline
\end{tabular}

Tabla 19. Potasio (mg/L).

\begin{tabular}{|lllll|}
\hline Trat. & $\mathbf{2 0 1 5}$ & $\mathbf{2 0 1 6}$ & $\mathbf{2 0 1 7}$ & Media \\
\hline T & 1820 & 1956 & 1585 & 1787 \\
\hline D & 2005 & 1983 & 1598 & 1862 \\
\hline Sig. & $*$ & & & \\
\hline
\end{tabular}

\section{Conclusiones}

La aplicación del despampanado al inicio de floración provocó una reducción notable de la producción de uva, aproximadamente un $32 \%$ de media interanual, respecto al testigo. Esta reducción de rendimiento fue debida completamente al menor número de racimos por cepa, un $31 \%$ inferior, derivado del despampanado, ya que el peso del racimo no mostró ningún efecto definido que hubiera podido compensar parcialmente la pérdida de cosecha. Las escasas variaciones anuales observadas en el número de bayas por racimo y en el peso de baya no contribuyeron en conjunto a modificar apreciablemente el peso de racimo.

El peso de madera de poda se vio claramente reducido a causa del despampanado, en un $16 \%$ de media, como consecuencia directa de la supresión de un $31 \%$ de los sarmientos. El aumento del peso de sarmiento causado por el despampanado, un $2 \overline{2 \%}$ de media, compensó parcialmente la reducción del número de pámpanos en cuanto al peso final de madera de poda. El índice de Ravaz se vio reducido por la mayor disminución de la producción de uva que del peso de madera de poda en el tratamiento sometido a despampanado.

La concentración de sólidos solubles mostró un aumento constante debido al despampanado, de $0.7^{\circ}$ brix de media interanual, ya que favoreció la relación hojas/frutos con respecto al testigo. $\mathrm{El} \mathrm{pH}$ varió ligeramente a favor del tratamiento de despampanado, excepto el año de sequía pronunciada, en que no hubo diferencia entre despampanado y testigo. La acidez total y el ácido tartárico no presentaron una tendencia interanual definida ni constante, mientras que el ácido málico mostró valores anuales con tendencia inversa entre tratamientos con respecto a la acidez total y el ácido tartárico, sin diferencias notables entre tratamientos. La concentración de potasio fue ligeramente superior en el tratamiento de despampanado que en el testigo. Los resultados relativos a azúcares, $\mathrm{pH}$ y potasio se corresponden con cierta intensificación del proceso de maduración de la uva, causada por la modificación favorable del ratio hojas/frutos debida al despampanado.

Esta intensificación de la maduración vislumbra la posible utilidad de esta operación en añadas en que pueda haber retraso en el proceso de maduración, con el fin de optimizar la fecha de vendimia y la calidad de la uva. No obstante, las implicaciones enológicas y productivas que puede conllevar la ejecución del despampanado han de ser consideradas conjuntamente, en función de los objetivos productivos y económicos pretendidos.

El desarrollo de este estudio ha sido posible gracias a la financiación del proyecto INIA RTA2014-00049-C05-01 y fondos FEDER, la ayuda del personal técnico de Grupo Yllera S.L. y de la Estación Enológica del Instituto Tecnológico Agrario de Castilla y León.

\section{Referencias}

[1] M.V. Alburquerque, R. Yuste, E. Barajas, J. Yuste, Vida Rural 310, 32 (2010)

[2] J. Yuste, A. Vicente, Phytoma 274, 116 (2015)

[3] J. Yuste, A. Vicente, D. Martínez-Porro, VI Congreso internacional CERVIM, La Laguna, Tenerife (España), 26-28 abril (2018)

[4] J. Yuste, D. Martínez, R. Yuste, La Semana Vitivinícola 3.438, 6 (2015)

[5] B.A. Bravdo. Acta Hort, (ISHS) 652, 119 (2004)

[6] L. Hidalgo, "Tratado de viticultura general",

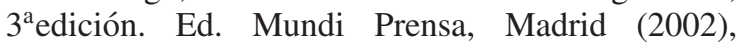
$1235 \mathrm{p}$.

[7] A. Palliotti, A. Cartechini, Acta Hort. 512, 111 (2000)

[8] D.S. Intrigliolo, J.R. Castel, Irrigation Science 29, 443 (2011)

[9] A. Calderón-Orellana, L. Mercenaro, K.A. Shackel, N. Willits, M.A. Matthews, Am. J. Enology and Viticulture 65, 354 (2014)

[10] M.V. Alburquerque, C. Arranz, E. Barajas, J. Yuste, Viticultura Enología Profesional 100, 21 (2005)

[11] R. Yuste, J. Nicolás, J. Yuste, Vida Rural 239, 22 (2006)

[12] M.C. Ramos, G.V. Jones, J. Yuste, Eur. J. Agronomy 70, 57 (2015) 